\title{
ARTIFICIAL INTELLIGENT RECRUITMENT SYSTEM
}

\author{
Siddhant Singh \\ Department of Computer Engineering \\ International Institute of Information Technology, \\ Pune, Maharashtra, India \\ Vishesh Waghmare \\ Department of Computer Engineering \\ International Institute of Information Technology, \\ Pune, Maharashtra, India
}

\begin{abstract}
Artificial Intelligence (AI) technology has revolutionized every aspect of personal as well as professional life by making everything smarter. AI for selection of suitable candidates is a developing category of HR automation devised to curtail or even abolish time engrossing tasks like manually screening resumes. The artificial intelligent recruitment system is devised to automate few tasks of the recruiting work flow, especially repetitive, high-volume tasks. Due to artificial intelligence in recruitment, data becomes easier to collect, access, and analyze. The advantage of adopting $\mathrm{AI}$ in the recruitment system is its capability to make use of data to institutionalize the practicality between the candidates experience, expertise, ability, competence and the requirements of the job. Another appealing benefit of AI in recruitment, still in the sourcing field can be found in the candidate matching part of the process. AI technology can be used to enhance the prospect experience, that is to exactly figure out what position and type of content your prospects are engrossed in. Among other things, NLP and AI can help on resume classification, ranking, deep extraction, identification and semi-automation in the recruitment process.
\end{abstract}

Keywords-Artificial Intelligence, Automated Recruitment, Human Resource, Talent Acquisition.

\section{INTRODUCTION}

In the staffing and recruitment industry, recruitment teams need to physically visit a particular campus or conduct a placement drive and deal with thousands of candidates personally. Employers work hard every day to source top talent and it's a challenge for them to communicate well with all their candidates. A huge amount of effort and management is needed for this process; also the process itself is tedious and time-consuming. Moreover, due to the recent outbreak of the novel corona-virus, the main priority has been shifted to the safety of the company staff. Interacting with a number of candidates from different places for a hiring drive cannot be a

\author{
Ishali Jadhav \\ Department of Computer Engineering \\ International Institute of Information Technology, \\ Pune, Maharashtra, India \\ Rajeev Ramesh \\ Department of Computer Engineering \\ International Institute of Information Technology, \\ Pune, Maharashtra, India
}

solution anymore. We must cut it down as much as possible. Perhaps, AI can save the day! In today's case scenario we can see AI can be effectively implemented in many different ways. $\mathrm{AI}$ has the potential to relieve them of this laborious task and make them focus on even better things. An $\mathrm{Al}$ recruiter will help to reduce the manpower and the tedious task to short-list the candidates. By making it completely data-managed, an AI Recruiter will typically produce more closely matched hires with higher retention rates keeping the procedure totally unbiased. It allows talent acquisition officers and hiring executives to make data- driven hiring decisions rather than decisions based on their gut feeling. Freelancers do not have enough manpower required to carry out selection processes. This tool will make it much easier and efficient to assign the project to the ideal person. This can be used for selecting students for various internships as well. Students applying for various posts and different skill sets will be selected according to the requirement. The following proposed solution can also be implemented using slack to keep a track of the skill sets of the employees working on a particular project so that if the project urgently requires a specific task to be done, the system will automatically recommend the employee.

\section{METHODOLGY}

\section{A. Proposed System}

The artificial intelligent recruitment system subsists of a Candidate Panel and Admin Panel. As the name suggests the candidate panel is for the applicants applying for a desired position, whereas the admin panel is for HR officers and recruiters. Both the panels are created with help of NodeRED, the database is Cloudant and various IBM Cloud services such as Personality Insights, IBM Discovery, are used for a hassle free recruitment process. This solution helps, recruiting teams to screen and select candidates, saving a huge amount of time. 


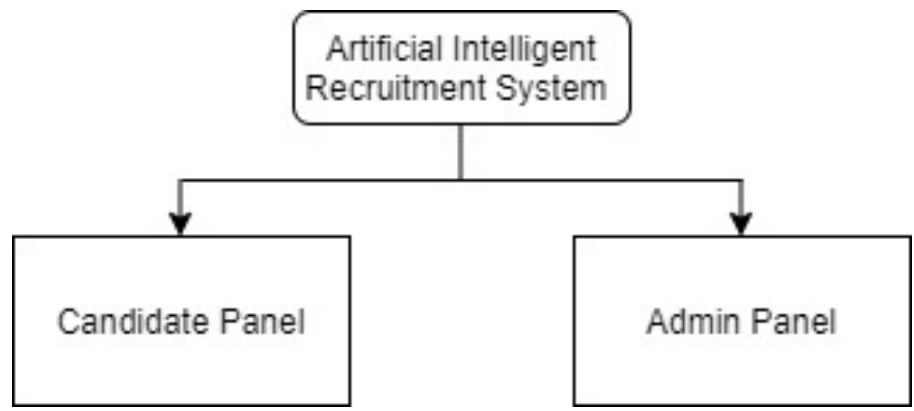

Figure 1: System Overview

\section{B. Candidate Panel}

The applicants have to undergo three steps for the system to successfully identify them as selected candidates. Each step has a touchstone, and by accomplishing those the candidate proceeds to the next step. Registration, Technical Round and Personality Assessment are the three rounds the candidate has to complete in order to place a position as selected candidate. The figure below explains the complete flow of the Candidate Panel. Figure 2 represents the candidate panel architecture.

\section{Registration}

All the applicants have to fill the registration form first. The details that the applicant fills gets saved in the database with a unique id. The recruiters and HRs further contact the applicants using these details filled by the applicant. The applicants are then asked to upload their resume/CV in .pdf format.

\section{Resume Scanning}

The resume/CV uploaded by the candidate are in .pdf format, therefore first text extraction is done. All the text extracted from the applicants resume/CV are then passed to the keyword mapping system, where the applicants resume is scanned and tested whether it meets the recruiters requirements or not. After the resume is excerpted by the system, the applicant is then further promoted to the next round. If the applicant's resume doesn't meet the recruiters requirement, the applicant is then asked to exit the system. Applicants selected from the registration round are now Shortlisted Candidates.

\section{Technical Round}

The shortlisted candidates further have a technical round which is conducted by a Chatbot which is trained to ask MCQs related to the designation the applicant is applying for. The Chatbot asks the shortlisted candidates various questions according to the designation they are applying for. In the proposed system the Chatbot is created using IBM Watson Assistant one of the IBM Cloud Service. The Chatbot awards marks to the shortlisted applicant according to the answer submitted by the applicant. There is a minimum passing score in the system for the applicant to proceed further for the next round. The passing criteria and the questions can be changed according to the recruiters' needs.

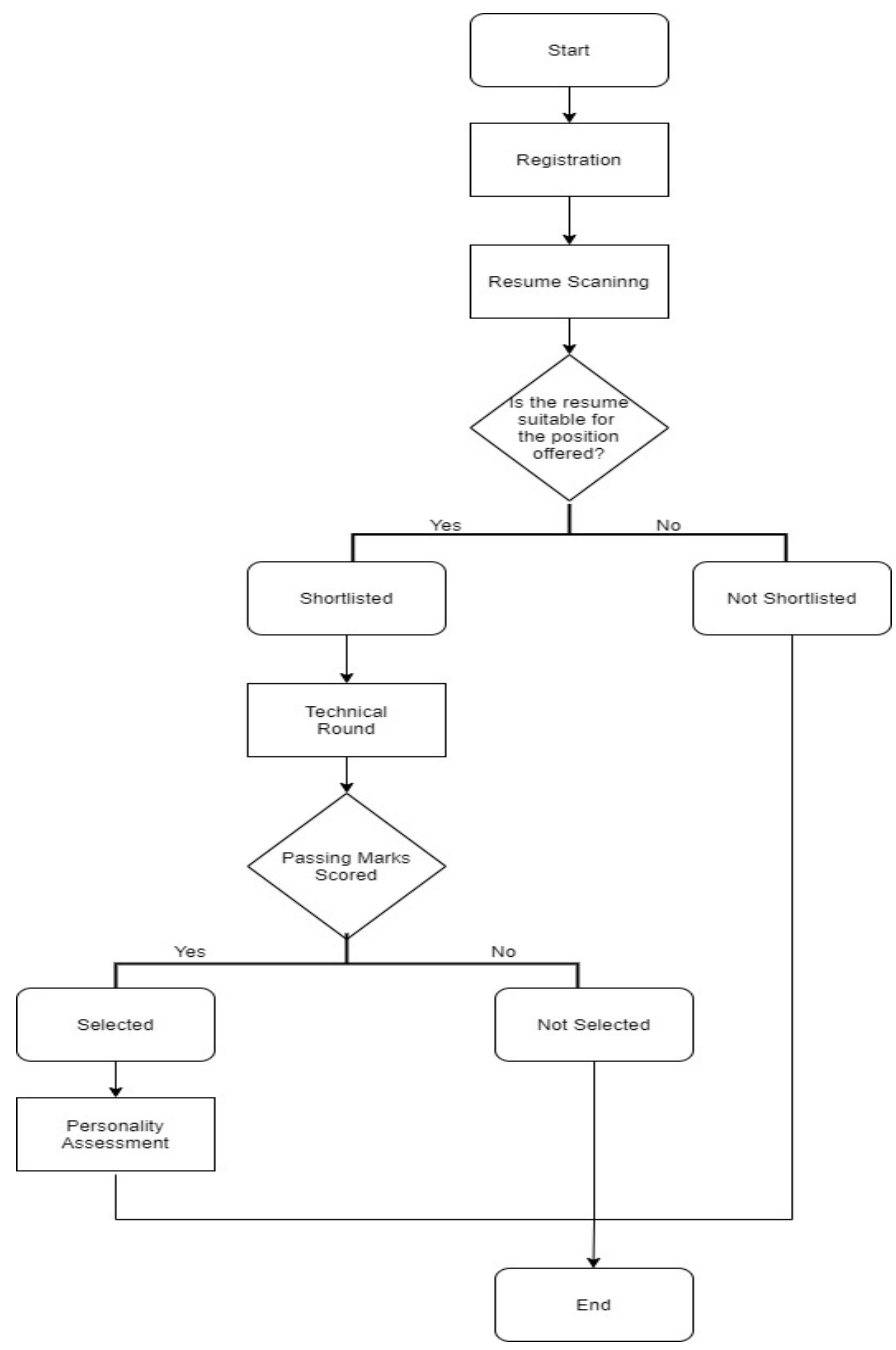

Figure 2: Candidate Panel Flowchart

\section{Personality Assessment}

In this round the applicant has to answer some questions with which the personality traits of the applicant can be found. By means of IBM Cloud Service - Personality Insights we can get the various personality traits of the selected candidate like emotions, attitude, behavior, nature, etc. The answers given by the selected candidates are further classified using aspects like openness, curiosity, practicality, integrity, cordiality, challenge, sympathy and certainty 


\section{Admin Panel}

After all the applicants from a particular institution or organization complete all the procedures. The details and results of all the candidates get saved in the database which is created using Cloudant. The recruiters get information of all the shortlisted as well as selected candidates by virtue of a designed dashboard. In the admin panel, the personality traits of the selected candidates are also displayed. The selected students are sorted as a result of the score the applicants scored in the technical round. For further judgement the recruiters can take help of the personality assessment test score. By means of the admin panel even though an applicant is not selected the recruiter can stay in contact with the applicant because of the details the applicant had filled in the registration form. Many not selected candidates never hear from their recruiters, so this factor in our system cloaks this flaw. Figure 3 represents the admin panel architecture.

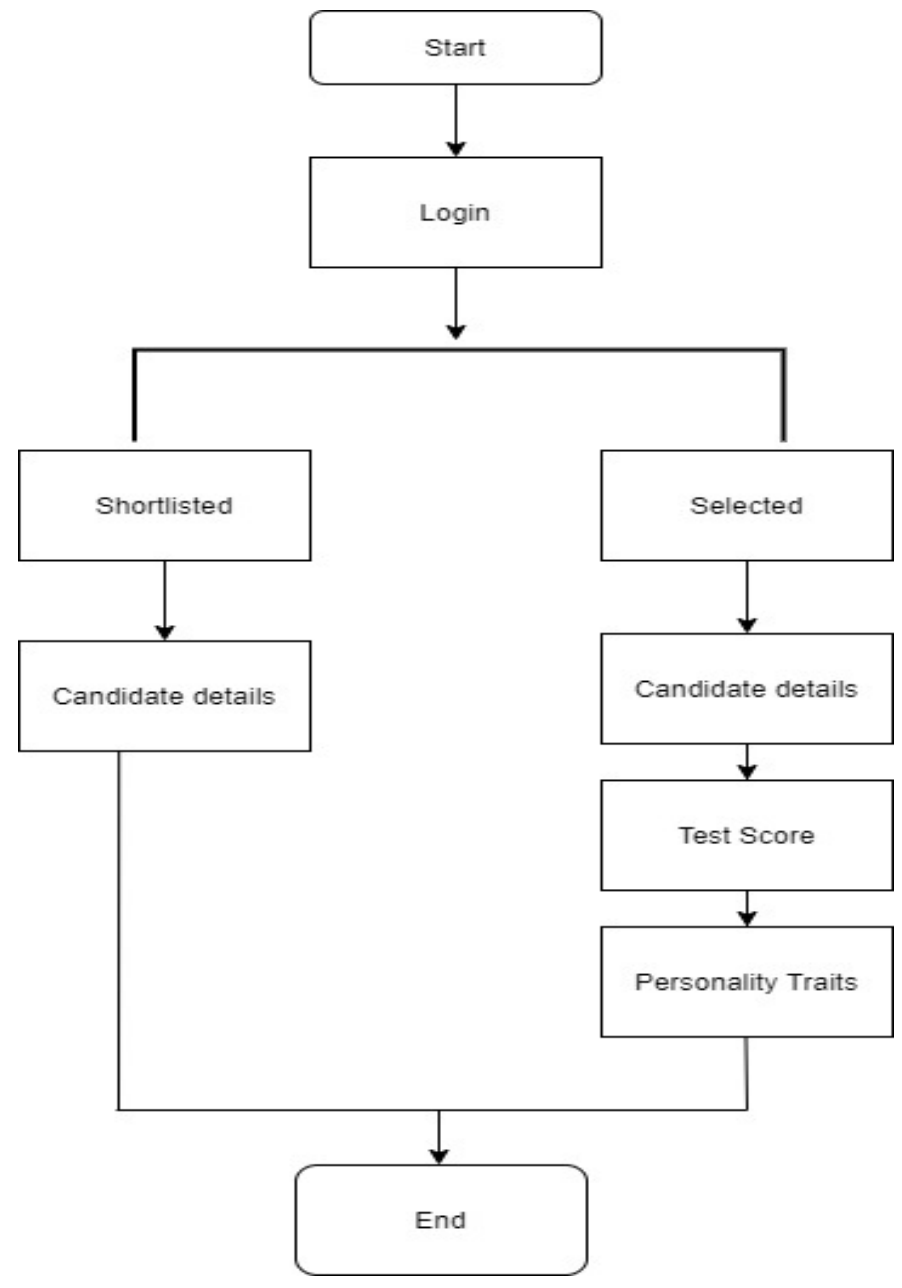

Figure 3: Admin Panel Flowchart

III. SOFTWARE CONFIGURATION

\section{Node-RED}

Node-RED is a programming tool for bringing together hardware devices, APIs and online services in advanced and engaging ways. It provides a browser-based editor that makes it effortless to wire together flows using the wide range of nodes in the palette that can be deployed to its run time in a single-click.

\section{PDF Hummus}

Node-RED node to be used to excerpt text from a .pdf file making use of hummusjs. This node splits text out of a PDF document making use of the npm module hummus js and the text extraction sample.

\section{Cloudant}

IBM Cloudant is a fully managed database service for hybrid multiple cloud applications built on open source Apache Couch DB with a fully compatible API. Sync data to any cloud or to the edge.

\section{IBM Watson Discovery}

Watson Discovery applies the latest breakthroughs in machine learning, including natural language processing capabilities, and is easily trained on the language of your domain.

\section{IBM Watson Personality Insights}

Predict personality characteristics, needs and values through written text. Understand your candidates' habits and preferences on an individual level, and at scale.

\section{IBM Watson Assistant}

Watson Assistant is a conversation AI platform that provides fast, straightforward and accurate interpretation to the query raised in the system.

\section{Programming Languages}

Node.js, HTML, CSS and Javascript were the programming languages used in the proposed system.

\section{RESULTS}

The proposed solution of an artificial intelligent recruitment system can be implemented at various circumstances. It can be implemented during layoffs so that the company will have only a preferred number of employees that are needed for the ongoing projects. College committees can implement this tool to select the required number of members that are fit to join that particular committee without investing a lot of their time. The artificial intelligent system allows recruiters and hiring officers to make data-driven hiring decisions rather 
than decisions based on their gut feeling. The system pulls fresh data consistently and retains and monitors every data point in one platform. It will pick up any pattern that was present earlier but hasn't been excluded by the algorithm yet. The proposed system supports a unified recruiting ecosystem.

\section{CONCLUSION AND FUTURE WORK}

The artificial intelligent recruitment system is reliable and is uncomplicated. The idea of this system can be used by any organization and institution and can be re-designed according to need by making some minor changes. The future scope of the proposed system is to create a model using machine learning algorithms from the data received from the selected applicants resume/CV. So, when the organization or institution needs an employee for a specific designation, the selection can be done according to the data of the resume by means of which the model was created. The model will help to predict the best suitable applicant according to the requirement and need of the organization or institution. Also, the organization will be successful in creating a workspace for their employees who have similar thinking and skills. This will not only help the organization to grow but also the employees will enjoy the work environment.

\section{REFERENCES}

[1] P. Cappelli, P. Tambe, and V. Yakubovich et al, (Nov.2018.), "Artificial intelligence in human resources management: challenges and a path forward," SSRN Electron. J.,

[2] E. Parry and V. Battista, "The impact of emerging technologies on work: a review of the evidence and implications for the human resource function," Emerald Open Res., vol. 1, p. 5, Jan. 2019.

[3] J. Hill, W. Randolph Ford, and I. G. Farreras, "Real conversations with artificial intelligence: a comparison between human-human online conversations and human- chatbot conversations," Comput. Human Behav., vol. 49, pp. 245-250, 2015.

4] A. Følstad and P. B. Brandtzæg, "Chatbots and the new world of HCI" Interactions, vol. 24, no. 4, pp. 38-42, 2017.

[5] B. Sheth, "Chat bots are the new HR managers,"Strateg. HR Rev., vol.17, no. 3, pp. 162163, Apr. 2018.

[6] K. Kuksenok and N. Praß, "Transparency in maintenance of recruitment chatbots" in Where is the
Human? Bridging the Gap Between AI and HCI Workshop , 2019, pp. 1-4.

[7] P. Nikhila1, G. Jyothi, K. Mounika3, M. C. Kishor, K.Reddy, and R.Murthy, "Chatbots using artificial intelligence,” J. Appl. Sci. Comput., vol. 6, no. 2, pp. 103-115, 2019.

[8] E. He, "Can artificial intelligence make work more human?,” HR Rev., vol. 17, no. 5, pp. 263-264, Nov. 2018 .

[9] A. DiRomualdo, D. El-Khoury, and F. Girimonte, "HR in the digital age: how digital technology will change HR's organization structure, processes and roles,"Strateg. HR Rev,vol.17,no.5,pp.234-242,Oct. 2018.

[10] J. Gikopoulos, “Alongside, not against: balancing man with machine in the HR function," Strateg. HR Rev., vol.18, no. 2, pp. 56-61, Apr. 2019.

[11] N. Nawaz, "A comprehensive literature review of the digital HR research field," Information Knowl. Manag., vol.7, no.4,pp.15-20, 2017.

[12] D. L. Stone, D. L. Deadrick, K. M. Lukaszewski, and R. Johnson, "The influence of technology on the future of human resource management," Hum. Resour. Manag. Rev., vol. 25, no. 2, pp. 216-231, Jun. 2015.

[13] Uday Kamath et al, (2019), Deep Learning for NLP and Speech Recognition, Springer, (pp. 141-174).

[14] Wadhwani, Preeti, and Saloni Gankar, (2017), "Intelligent Virtual Assistant Market Size \& Share: Global Report 2024.” Global Market Insights, Inc., www.gminsights.com/industryanalysis/intelligentvirtual- assistant-iva-market.

[15] Matthews, K. (2019). 5 Ways Data Science Is Changing Financial Trading. Retrieved 21 April 2020, from https://towardsdatascience.com/5waysdata- science-is-changing-financialtradingb7404d62ed6d 